\title{
Event reconstruction performance with the GAPS experiment.
}

\author{
Riccardo Munini ${ }^{\star a, b}$, M. Boezio ${ }^{a, b}$, P. von Doetinchem ${ }^{c}$, N. Marcelli ${ }^{d}$, A. Stoess ${ }^{c}$, E. \\ Vannuccini ${ }^{e}$ \\ ${ }^{a}$ INFN, Sezione di Trieste, I-34149 Trieste, Italy \\ ${ }^{b}$ IFPU - Institute for Fundamental Physics of the Universe, I-34014, Trieste, Italy \\ ${ }^{c}$ University of Hawai'i at Manoa, Honolulu, USA \\ "INFN, Sezione di Roma "Tor Vergata", I-00133 Rome, Italy \\ e INFN Sezione di Firenze, I-50019 Sesto Fiorentino FI, Italy \\ E-mail: riccardo.munini@ts.infn.it,mirko.boezio@ts.infn.it, \\ philipvd@hawaii.edu, nadir.marcelli@roma2.infn.it, \\ stoessl@hawaii.edu, elena.vannuccini@fi.infn.it
}

\begin{abstract}
The General Antiparticle Spectrometer (GAPS) is an Antarctic balloon-borne detector designed to measure the low energy $(<0.25 \mathrm{GeV} / \mathrm{n})$ antideuteron cosmic ray component as a distinctive signal from dark matter annihilation or decay in the Galactic halo.

The detector consists of a tracker, made up by ten planes of lithium-drifted silicon $\mathrm{Si}(\mathrm{Li})$ detectors, surrounded by a plastic scintillator time-of-flight system.

GAPS uses a novel particle identification method based on exotic atom capture and decay with emission of pions, protons and atomic $\mathrm{X}$-rays from a common annihilation vertex. The resulting "nuclear star" topology provide sufficient rejection power to suppress any non-antiparticle cosmic ray background.

Here we present results for a preliminary reconstruction algorithm that has been tested on simulated events. The GAPS simulation software is based on GEANT4 and fully reproduce the detector geometry. Various technique like Kalman filter, Hough-3D transform, Least Squares minimization were combined in order to precisely reconstruct the topology of the "nuclear star". The performance of the final algorithm in reconstructing the annihilation vertex position and the secondary pions will be discussed.
\end{abstract}

36th International Cosmic Ray Conference -ICRC2019-

July 24th - August 1st, 2019

Madison, WI, U.S.A.

${ }^{*}$ Speaker. 

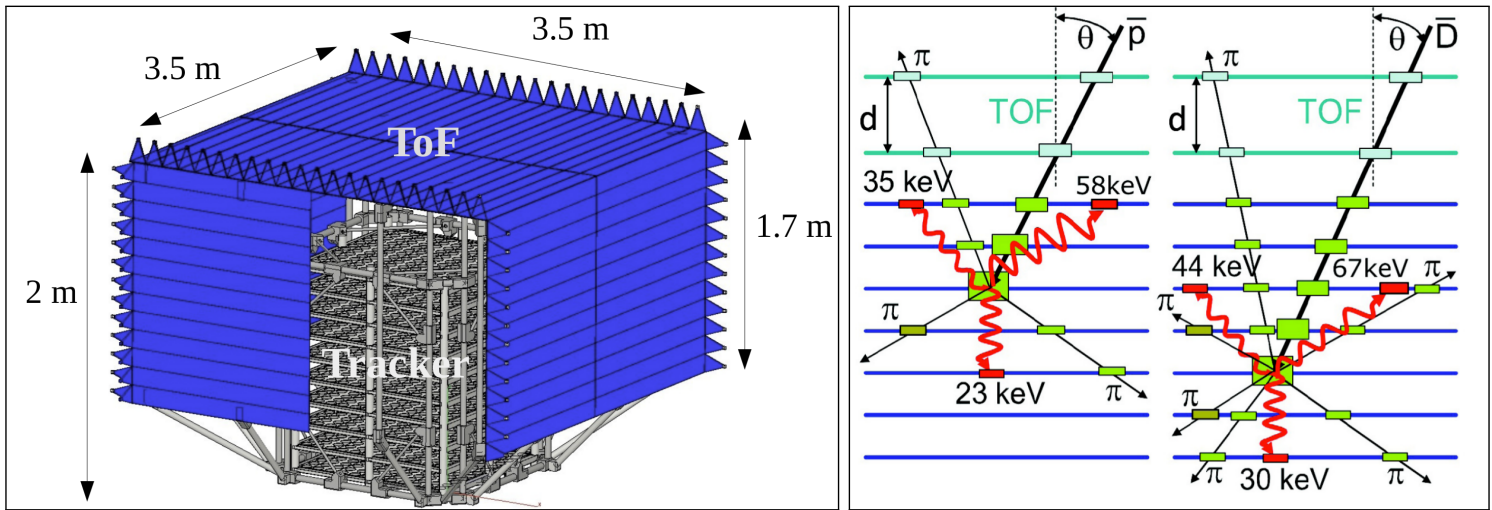

Figure 1: Left panel: schematic view of the GAPS apparatus. Right panel: event topology expected from an antiproton (left) and an antideuteron (right) of the same initial velocity.

\section{The GAPS detector}

The General AntiParticle Spectrometer (GAPS) is a balloon flight experiment designed for indirect dark matter search with low energy $(<0.25 \mathrm{GeV} / \mathrm{n})$ cosmic ray antideuterons. The first GAPS flight is scheduled for the austral summer of 2021-2022. Figure 1 (left panel) shows a schematic view of the GAPS apparatus. The core is a tracker made of 10 planes of cylindrical $\mathrm{Si}(\mathrm{Li})$ detectors $(2.5 \mathrm{~mm}$ thick $\times 10 \mathrm{~cm}$ diameter) segmented in 8 strips [1]. On each plane the SiLi cylinders are arranged in a $6 \times 6$ array of module with $4 \mathrm{Si}(\mathrm{Li})$ detectors each. Precise reconstruction of the event topology is the main goal of the tracker. These ten planes are surrounded by an inner and an outer time of flight (Tof) composed by 196 bars of plastic scintillator. The Tof will provide the time information need to reconstruct the particle velocity and to trigger the acquisition [2]. For more details on the scientific motivations and the GAPS apparatus see [3].

The GAPS detection technique will be complementary with respect to the magnetic spectrometers like AMS02, PAMELA or BESS. Antinuclei which have been sufficiently slowed down by $\mathrm{dE} / \mathrm{dx}$ losses can form an exotic atom replacing a valence electron in a Si atom. Subsequently the exotic atom decays with a series of atomic transition emitting X-rays of specific energy. Finally, when the primary reach the nucleus, it annihilates producing secondary particles (pions and protons) from a common vertex. The expected topology from an antiproton (left) and an antideuteron (right) annihilation is showed on Figure 1 (right panel).

In order to identify antideuteron over the much more abundant antiproton component a rejection of about $10^{6}$ is necessary. Antiproton-antideuteron identification will be performed combining several quantities like the range and slow down profile of the primary, the energy of the X-rays, the number of secondaries. Especially important are the penetration depth and the slow down of the primary thus is very important to reconstruct with high precision the velocity and the energy losses of the primary as well as the position of the vertex.

Here the status and the performances of a preliminary reconstruction algorithm will be discussed. The algorithm has been developed within the framework of the GAPS simulation software which fully reproduces the instrument geometry and materials by means of GEANT4. A set of antiprotons and antideuterons were simulated to perform the study. The raw simulated data were 


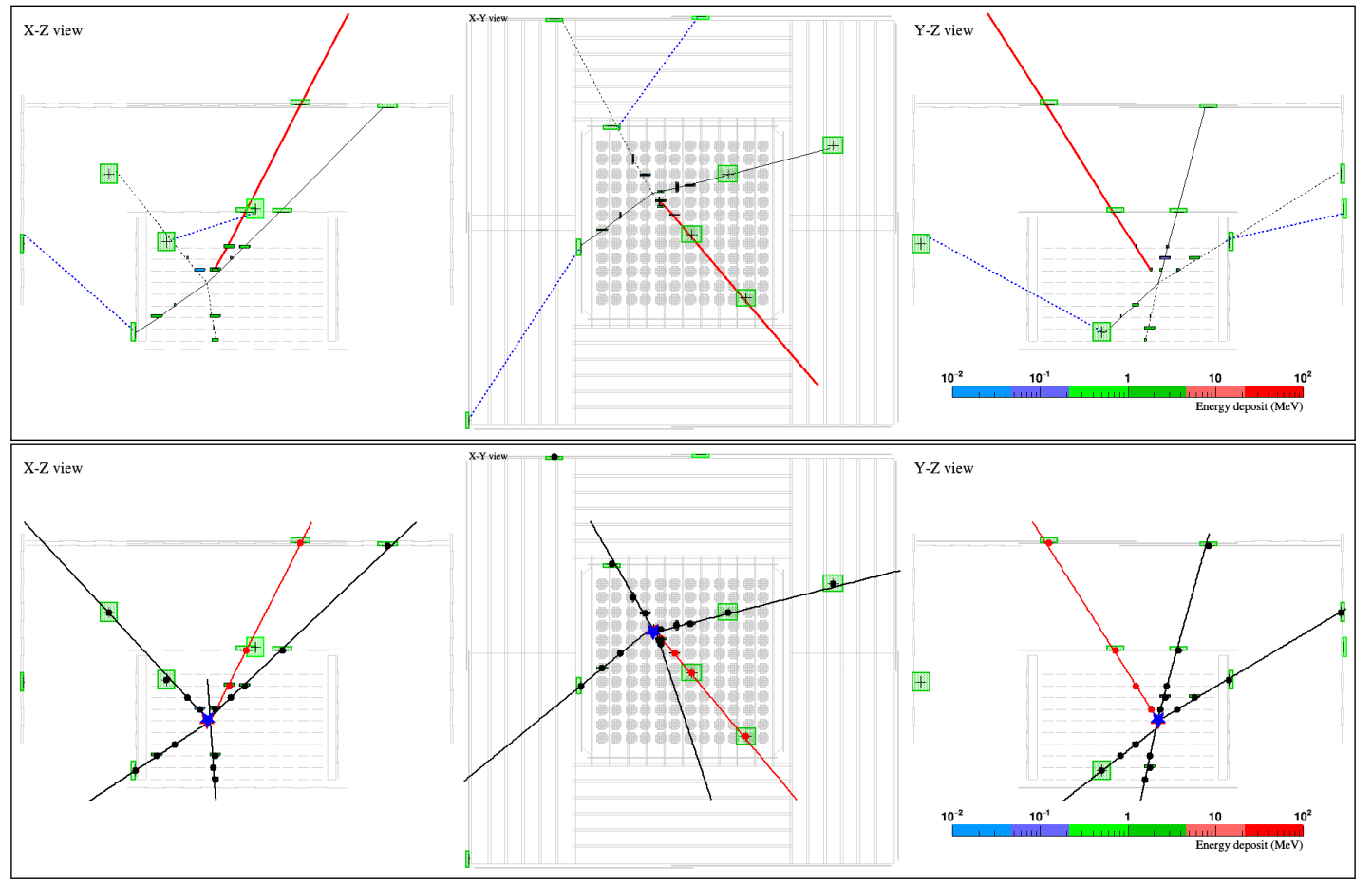

Figure 2: Top panel: simulated antiproton annihilation inside the GAPS apparatus. Solid lines represent MC particle trajectory: antiproton (red), pions (black) and electrons (blue). Colored blocks represent particle energy deposition in the active volumes. Bottom panel: reconstructed antiproton event perform with the global algorith. The red line is the reconstructed primary and the black line are the secondaries. The circle point are the hit associated to the reconstructed track. The red and the blue stars represent respectively the $\mathrm{MC}$ and the reconstructed vertex position.

digitized in order to introduce a realistic instrument response for the time and the energy measurements. A time resolution of $\sigma_{t}=500$ ps was considered while the energy resolution in this first phase was considered constant at $5 \%$.

\section{Event reconstruction}

The event reconstruction must provide the best estimation of the quantities necessary to perform the antinuclei identification:

- $\beta$ (defined as the ration of the velocity and the speed of light) and energy losses of the primary,

- number of secondaries;

- position of the annihilation vertex;

As explained in the previous section the annihilation topology is a star of secondary tracks originating from a common vertex. The reconstruction procedure is divided in three steps:

1. Primary antinucleus reconstruction; 
2. Secondary particle reconstruction;

3. Annihilation vertex reconstruction;

The reconstruction algorithm was developed studying Monte Carlo (MC) events which annihilates inside the tracker volume. From hereafter a straight line in the three dimensional space is defined with the parametric representation $\mathbf{v}=\mathbf{a} \cdot t+\mathbf{b}$ with $\mathbf{v}=(x, y, z)$ the spatial coordinates $\mathbf{a}=\left(a_{x}, a_{y}, a_{z}\right)$ the line direction and $\mathbf{b}=\left(b_{x}, b_{y}, b_{z}\right)$ the anchor point. The vector $\mathbf{b}$ is normalized so there are only 5 parameters.

\subsection{Primary reconstruction}

A track following approach has been used to reconstruct the primary track. It is assume that the primary will be the first particle to hit the outer and the inner Tof. Consequently the reconstruction begins selecting the hit with the smaller time in the inner and in the outer Tof. Since adjacent Tof paddles superimpose for few millimeter, about $20 \%$ of the primaries have multiple hit (up to four) in the inner and/or in the outer Tof. Adjacent Tof hit are associated to the primary if their absolute time difference (respect to the smaller time) is less than $1 \mathrm{~ns}\left(\sim 2 \cdot \sigma_{t}\right)$. In this way, the possibility to associate hit originated by the passage of a secondary is drastically reduced. At this point a least square minimization on the hit positions is performed to determine the primary trajectory. This trajectory is then extrapolated to search, if present, other primary hits. Two consistency checks are performed to the tracker hit in order to be associated to the primary:

1. Spatial consistency: hit are selected only if they are spatially consistent with the trajectory determined with the Tof. The impact position on the first tracker plane crossed by the projected track $\left(x_{\text {intercept }}\right)$ is calculated with the associated uncertainty $\left(\delta_{\text {intercept }}\right)$. The distance $d$ between the hit position and $x_{\text {intercept }}$ is then calculated. If $d<3 \cdot \delta_{\text {intercept }}$ the hit is selected.

2. Energy consistency: if the spatial check is satisfied, the energy deposit associated to the hit has to be consistent with a slow down particle. Consequently the energy $E$ of the hit is required to be greater than $a \cdot E_{i-1}$ where $E_{i-1}$ is the energy associated to the previous primary hit. In order to take into account for the fluctuations in the ionization energy losses the factor $a$ is taken equal to 0.8 .

If more than one hit satisfies these requirements, the hit with the highest energy is selected. Improvments to the algorithm are under study. For example in this case the decision on the energy to select will be improved by calculating the total amount of material traversed by the particle up to that plane and estimating the most probable energy release in that volume. Finally, if a hit has been associated, a least square minimization is performed again including the new position to refine the trajectory. This process is iterated for each tracker plane crossed by the extrapolated prima. The quality of the reconstructed trajectory is represented by the reduced Chi2 obtained from the minimization process. The beta of the primary is determined by a least square fit combining the flight path and times derived from the selected inner and outer Tof hits.

\subsection{Secodary reconstruction}

Two methods has been tested to reconstruct the secondary tracks. 


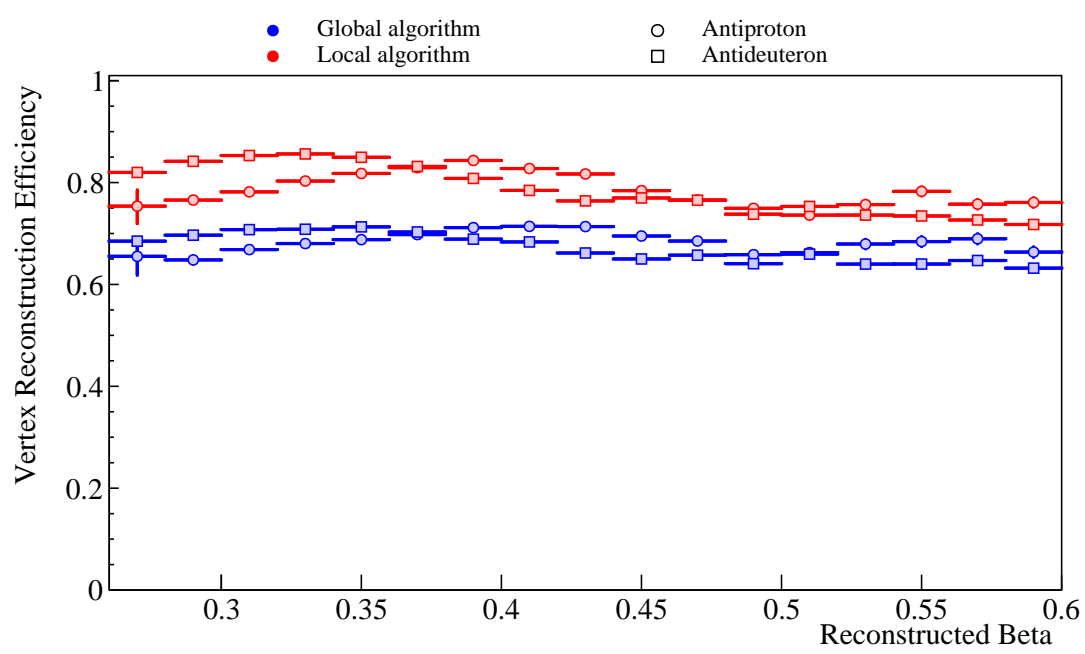

Figure 3: Vertex reconstruction efficiency as a function of the reconstructed primary beta. Blue points represent the performance of the Hough transform on antiproton (circles) and antideuteron (squares). Red points represent the efficiency of the Fit Star algorithm.

1. Global algorithm: this algorithm based on the Hough transform was adapted from [4] and is very powerful to associate aligned points in a three dimensional space. The global algorithm searches in the parameter space the set of $(\mathbf{a}, \mathbf{b})$ which best describes a set of aligned point in the dimension space. The most relevant parameter of the algorithm is the segmentation of the parameter space which has to be adequately chosen depending on the spatial resolution. Few modification were applied to the original code in order to specifically study the annihilation topology. A constrain is applied by forcing the algorithm to detect straight line which are distant no more than few centimeter from the primary track. This constrain is very important since it reduces drastically the possibility to associate points which by chance are aligned on a straight line but do not originate from the annihilation vertex. The multiple scattering is the main limitation to the performances of the algorithm since the hits produced by the passage of a particle are not aligned on a straight line. The Hough transform is applied recursively: when a set of hits has been associated to a track they are removed and the algorithm applied again. A least square minimization is finally performed on each set of selected positions in order to estimate the secondary trajectories.

2. Local algorithm: this procedure performs a spatial scan along the primary direction starting from the position of the last hit. The scan is carried out with a segmentation of $0.5 \mathrm{~cm}$ for a length of $50 \mathrm{~cm}$. At each step an angular scan is performed propagating $i=5000$ trajectory isotropically distributed over the solid angle. For each direction which intercept at least $n>1$ hits the quantity $q$ is defined as $q=\frac{N}{n}$ where $N$ is the total number of hits in the event. Once the spatial scan is terminated the quantity $Q=\prod_{i} q_{i}$ is defined summing over all the selected directions. The minimum value of the quantity $\log Q$ corresponds to the position along the primary track which maximize the number of intercepted points. The points associated to each trajectory having at least two points are finally used for a least square minimization in order to refine the particle trajectory. 
The global and the local algorithms are applied independently on the events. The performances of the two algorithms will be discussed in Section 3. When at least one secondary is reconstructed the annihilation vertex position is estimated.

\subsection{Vertex estimation}

The reconstructed tracks are finally combined in order to estimate the vertex position. The vertex is determined as the point in the space which minimize the weighted sum of the distances from the tracks. A first estimation of the vertex is perform with all the tracks (primary + secondaries). Then, to refine the vertex estimation, a series of cuts are applied to reject tracks with bad quality or which are not consistent with the annihilation topology. A track is rejected if:

- $\mathrm{n}_{h i t}<N$ with $\mathrm{n}_{h i t}$ the number of the hit associated to a track;

- $\mathrm{Chi} 2_{\text {track }}>C$ with Chi $2_{\text {track }}$ the chi square associated to the tracks;

- $\mathrm{d}_{\text {vertex }}>D \quad$ with $\mathrm{d}_{\text {vertex }}$ the distance of the track from the vertex in $\mathrm{mm}$;

If one or more tracks are rejected the vertex estimation is performed again. The value (N,C,D) has been calibrated to provide the best balance among the vertex resolution and a sufficiently high reconstruction efficiency.

A convolutional neural network is also under study for the vertex reconstruction. The advantage of such approach is the fast execution time and the high reconstruction efficiency. The key point is the training of the algorithm which has to be properly done in order to achieve competitive performances with the classical approach.

\subsection{Reconstructed event}

Figure 2 shows a simulated antiproton with $\beta=0.55$ which annihilates inside the tracker. The top panel displays the MC tracks. The primary antiproton (red line) stops in the fourth tracker plane and four secondary pions (black solid line $\pi^{+}$and dotted line $\pi^{-}$) exit from the annihilation vertex. The colored boxes represent the energy deposited in the active volumes by the particles. The low panel of Figure 2 shows the reconstructed event. The red dots are the hit associated to the primary and the red line is the primary trajectories. The black points are the hit associated to the secondaries and the black lines the corresponding trajectory. The reconstructed vertex is represented by the blue star which almost completely overlap with the red star which is the annihilation position from MC

\section{Reconstruction performances}

The performances of the reconstruction algorithm have been tested on simulated antiprotons and antideuterons. The relevant features are listed below:

- Computational time: the global algorithm transform is relatively fast taking around $0.2-0.5$ seconds to process an event. The local algorithm is significantly slower due to the fine scanning performed along the primary trajectory. An event typically requires about $10-15$ seconds to be processed with the local algorithm. 

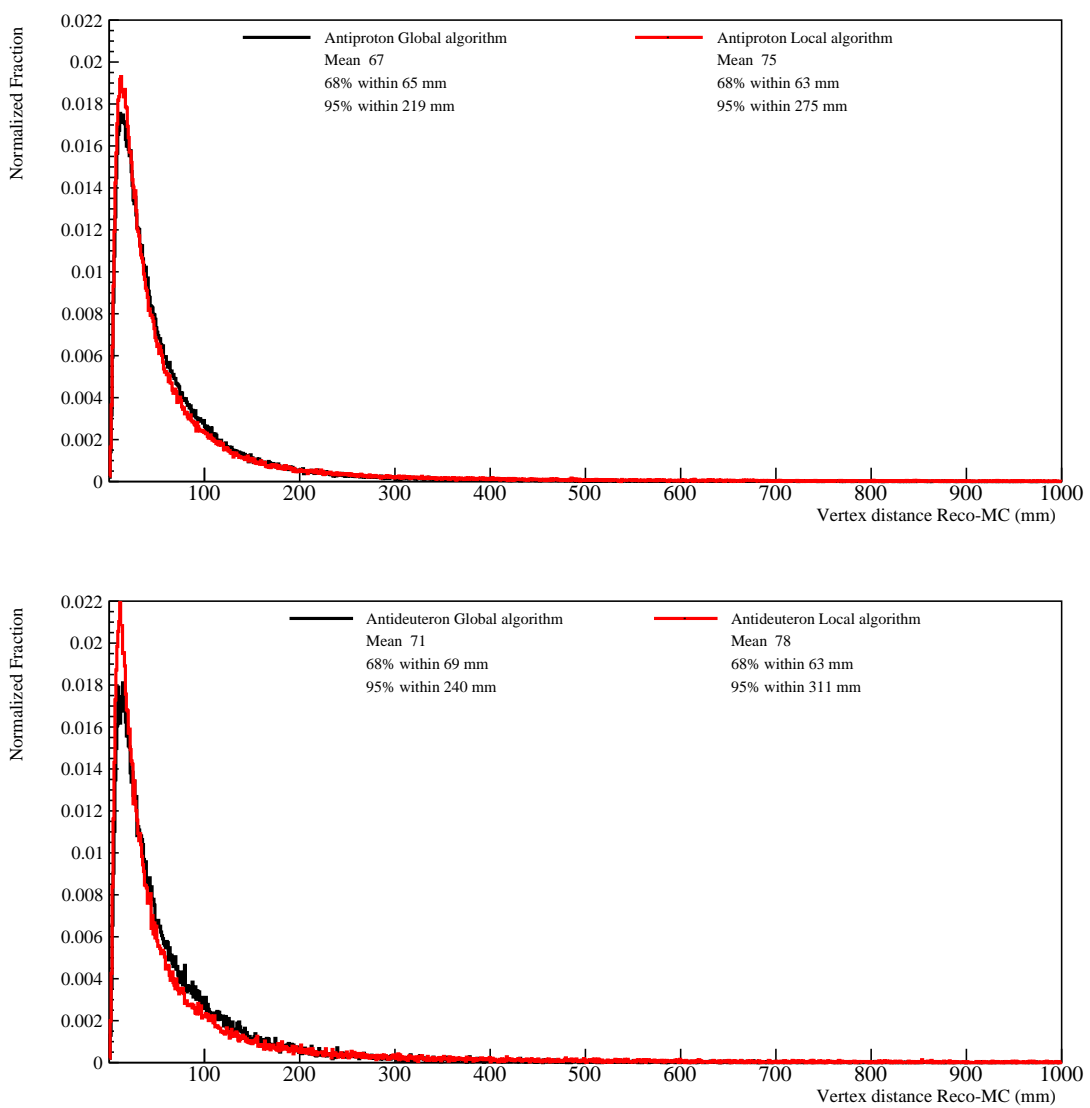

Figure 4: The resolution of the reconstructed vertex for simulated antiprotons (top panel) and antideuterons (bottom panel). The distribution is the distance between the MC and the reconstructed vertex position.

- Reconstruction efficiency: the vertex reconstruction efficiency has been estimated from a sample of events which annihilate inside the tracker volume. An event is reconstructed if at least two tracks are reconstructed and the vertex minimization converges. The result present here were obtained using $N=2, C=3, D=200$. In Figure 3 the vertex reconstruction efficiency as a function of the primary beta is shown. Blue points (circle antiprotons, square antideuterons) represent the efficiency for events reconstructed with the global algorithm while the red points represent the efficiency of the local algorithm. The efficiency of the local algorithm is slightly higher with respect to the global algorithm. In fact the local algorithm finds on average more secondary tracks being the search performed radially from a position relatively close to the vertex. This represent a very strong constrain since it reduces significantly the dimension of the parameter space to analyze. The global algorithm, even with the vertex constrain, is more general and has a larger parameter space to analyze increasing the probability to wrongly associate points hence resulting in a lower efficiency due to the quality cut.

- Vertex resolution: Figure 4 shows the distance in mm between the Monte Carlo and the reconstructed vertex position for simulated antiprotons (top panel) and antideuterons (bottom 
panel). In red the distribution for events reconstructed with the local algorithm while in black the events reconstructed with the global algorithm. All the distributions peak at around $15 \mathrm{~mm}$ which represent the intrinsic limit due to multiple scattering and the hit position resolution. The local algorithm distribution is slightly more peaked with respect to the global one but has also broader tail. The tail of these distributions has to be reduced as much as possible, for example by requiring more stringent quality cut. The level at which the tail has to be reduce in order to reach the antiproton rejection necessary to the antideuteron identification is currently under study.

\section{Conclusion}

The GAPS experiment will search for antideuteron in cosmic rays as a distinctive signal from dark matter annihilation. The detection technique based on the exotic-atom decay and annihilation will provide the $10^{6}$ antiproton rejection necessary for such challenging measurement. To reach this performances, the topology of the event must be precisely reconstructed. Here, a preliminary reconstruction algorithm has been presented. A combination of global and local algorithms has been combined in order to precisely reconstruct the annihilation topology. A high reconstruction efficiency ranging between $65 \%$ and $85 \%$ is obtained having a vertex resolution peaked at $15 \mathrm{~mm}$. The antinuclei identification with the reconstructed events is still under study and will drive future development and refinement of the reconstruction algorithm.

\section{Acknowledgments}

This work is supported in the U.S. by NASA APRA grants (NNX17AB44G, NNX17AB45G, NNX17AB46G, and NNX17AB47G), in Japan by JAXA/ISAS Small Science Program FY2017, and in Italy by Istituto Nazionale di Fisica Nucleare (INFN) and by the Italian Space Agency through the ASI INFN agreement n. 2018-28-HH.0: "Partecipazione italiana al GAPS - General AntiParticle Spectrometer". R.A. Ong receives support from the UCLA Division of Physical Sciences. K. Perez receives support from the Heising-Simons Foundation and Alfred P. Sloan Foundation. F. Rogers is supported through the National Science Foundation Graduate Research Fellowship under grant 1122374. P. von Doetinchem receives support from the National Science Foundation under award PHY-1551980. H. Fuke receives support from JSPS KAKENHI grants JP26707015, JP17H01136, and JP19H05198. M. Kozai receives support from JSPS KAKENHI grant JP17K14313. S. Okazaki receives support from JSPS KAKENHI grant JP18K13928. Y. Shimizu receives support from Sumitomo Foundation grant.

\section{References}

[1] Field, R., et. al., Large-area $\mathrm{Si}(\mathrm{Li})$ detectors for $\mathrm{X}$-ray spectrometry and particle tracking in the GAPS experiment, arXiv:1906.00054, 2019

[2] Quinn, S., et. al., Recent Progress on the GAPS Time of Flight System, ICRC 2019

[3] Bird, R., et. al., GAPS: Searching for Dark Matter using Antinuclei inCosmic Rays, ICRC 2019

[4] Dalitz, C., et. al., Iterative Hough Transform for Line Detection in 3D Point Clouds, IPOL 2017 